\title{
The prediction of response to immunotherapy in non-small cell lung cancer patients by 18F-FDG PET/CT
}

\author{
Laura Evangelista \\ Nuclear Medicine Unit, Department of Medicine-DIMED, University of Padova, Padua, Italy \\ Correspondence to: Laura Evangelista. Nuclear Medicine Unit, Department of Medicine-DIMED, University of Padua, Via Giustiniani, 235128 \\ Padua, Italy. Email: laura.evangelista@unipd.it. \\ Provenance: This is an invited article commissioned by the Section Editor Jun Zhou (Department of Nuclear Medicine, Zhongshan Hospital, Fudan \\ University, Shanghai, China). \\ Comment on: Jreige M, Letovanec I, Chaba K, et al. 18F-FDG PET metabolic-to-morphological volume ratio predicts PD-L1 tumour expression and \\ response to PD-1 blockade in non-small-cell lung cancer. Eur J Nucl Med Mol Imaging 2019;46:1859-68.
}

Submitted Sep 09, 2019. Accepted for publication Oct 08, 2019.

doi: $10.21037 /$ jtd.2019.10.19

View this article at: http://dx.doi.org/10.21037/jtd.2019.10.19

The role of FDG PET/CT in patients scheduled or undergoing immunotherapy is still unclear, particularly in those affected by non-small cell lung cancer (NSCLC). Recent papers have been published about this topic (1-5), drawing some preliminary indications. The recent paper by Jreige et al. (6), published in the European fournal of Nuclear Medicine and Molecular Imaging, tested a new PET biomarker able to predict the response to immunotherapy in a group of 49 patients with NSCLC. The authors found that a new variable called metabolic-to-morphological volume ratio (MMVR) correlated with the presence of necrosis and the PD-L1 expression in NSCLC that are both biomarkers for the responsiveness to immunotherapy. MMVR was obtained from a ratio between metabolic and morphological tumor volume: a high MMVR was indicative of a low PD-L1 expression, conversely a low MMVR was associated with a high PD-L1 expression.

In the study by Jreige et al. (6) emerged another important point: the presence of a necrotic lesion, assessed in terms of low MMVR is associated with high PD-L1 expression and therefore with a major responsiveness to immunotherapy. In fact, necrosis can trigger the inflammation thus facilitating the influx of $\mathrm{T}$ cells and therefore the upregulation of PD-L1 (7). The presence of hypoxia in the tumor, is associated with a low vessel density and with high GLUT-1 expression, it means by a high FDG uptake. In the study by Koh et al. (8), in 164 patients with lung adenocarcinoma, the authors found that PET parameters, such as SUVmax, TLG and MTV were significantly higher in patients with necrosis as compared to those without necrotic area. These results are complementary to the data from Jreige et al. (6).

Some considerations emerged from the abovementioned studies and therefore about tumor aggressiveness. First, it is characterized by a high FDG uptake, due to an increase in tumor growth and glucose metabolism. Second, the rapid tumor growth is associated with a low microvessel density and therefore with the appearance of a central photopenic area surrounded by intense FDG uptake suggestive of central necrosis. A high FDG uptake, in terms of SUVmax, in the lung lesion has already been correlated with the presence of a high PD-L1 expression and therefore with a major responsiveness to immunotherapy, in more than 500 patients (4). Moreover, high SUVmax at PET/CT, smoking and the presence of pleural invasion are all predictors of $\mathrm{PD}-\mathrm{L} 1$ protein expression in patients with lung cancer, especially NSCLC. Nevertheless, Jeirge et al. (6) did not find any correlations between SUVmax and PD-L1 expression or immunotherapy response in their experience, probably due to a small number of enrolled patients.

In fact, also Grizzi et al. (1) stated that the SUVmax of the primary lesion was able to predict the immunotherapy failure in 27 patients with NSCLC. Similarly, Evangelista et al. (3) showed that the sum of the semiquantitative data by FDG PET/CT was able to anticipate the progression during immunotherapy, especially in female patients with 
Table 1 A list of biomarkers for the assessment of response to immunotherapy

\begin{tabular}{|c|c|c|}
\hline $\begin{array}{l}\text { Imaging and non imaging } \\
\text { biomarkers }\end{array}$ & Pros & Cons \\
\hline \multicolumn{3}{|l|}{ Imaging } \\
\hline Radiological (mainly CT) & $\begin{array}{l}\text { - Specific criteria for the evaluation of response } \\
\text { to therapy }\end{array}$ & $\begin{array}{l}\text { - immune-related adverse events can be falsely } \\
\text { interpreted }\end{array}$ \\
\hline \multirow[t]{2}{*}{ FDG PET/CT } & $\begin{array}{l}\text { - Metabolic information may early predict the } \\
\text { response }\end{array}$ & $\begin{array}{l}\text { - FDG is not specific (high uptake also in inflamed } \\
\text { cells) }\end{array}$ \\
\hline & & $\begin{array}{l}\text { - The absence of specific criteria for the evaluation } \\
\text { of therapy }\end{array}$ \\
\hline \multirow{2}{*}{$\begin{array}{l}\text { Blood samples/tumor tissue } \\
\text { (multiple biomarkers*) }\end{array}$} & $\begin{array}{l}\text { - The ability to test the heterogeneity of tumor } \\
\text { tissue }\end{array}$ & - Variability among institutions \\
\hline & & - Tests' validation \\
\hline
\end{tabular}

*, PD-L1 expression, DNA mismatch repair (MMR) deficiency, the degree of cytotoxic T cell infiltration, mutational signature, antigen presentation defects, interferon signaling, tumor aneuploidy, T cell gene expression signatures, and the microbiota; CT, computed tomography.

\section{NSCLC.}

The responsiveness to immunotherapy can be assessed by using different methodologies. The most common are radiological imaging (i.e., contrast enhanced CT), but also other biomarkers have been tested. Table 1 reported a list of instruments that can be used. As illustrated some pros and cons have been reported for each biomarker. The most important is the absence of a standardization for many of them (both imaging and non-imaging), except for PDL1. However, PD-L1, as a single biomarker has some limitations, such as the influence due to prior therapies and the dependence to PI3K/AKT pathway. Therefore, PD-L1 expression cannot be considered exhaustive for the prediction of response to immunotherapy. The association of imaging and non-imaging biomarkers would be useful for reinforce the definition of responders or non-responders. Based on the last evidences (9), we can image an association of neutrophils/leukocytes ratio (NLR) with tumor mutation burden (TMB), lactate dehydrogenase (LDH) and imaging (metabolic or morphology) able to appropriately distinguish responders from non-responders. However, to date no information are available.

The main question is: is MMVR really as a new PET marker for the assessment of response to immunotherapy? In my opinion, it should be considered one of PET parameters that can help the clinicians to assess the potential responsiveness to immunotherapy in NSCLC patients, in concert with other non-imaging biomarkers.

\section{Acknowledgments}

None.

\section{Footnote}

Conflicts of Interest: The author has no conflicts of interest to declare.

Ethical Statement: The author is accountable for all aspects of the work in ensuring that questions related to the accuracy or integrity of any part of the work are appropriately investigated and resolved.

\section{References}

1. Grizzi F, Castello A, Lopci E. Is it time to change our 
vision of tumor metabolism prior to immunotherapy? Eur J Nucl Med Mol Imaging 2018;45:1072-5.

2. Ciarmiello A, Fonti R, Giovacchini G, et al. Imaging of immunotherapy response in non-small cell lung cancer: challenges and perspectives. Clin Transl Imaging 2018;6:483-5.

3. Evangelista L, Cuppari L, Menis J, et al. 18F-FDG PET/ CT in non-small-cell lung cancer patients: a potential predictive biomarker of response to immunotherapy. Nucl Med Commun 2019;40:802-7.

4. Takada K, Toyokawa G, Okamoto T, et al. Metabolic characteristics of programmed cell death-ligand 1-expressing lung cancer on $18 \mathrm{~F}$-fluorodeoxyglucose positron emission tomography/computed tomography. Cancer Med 2017;6:2552-61.

5. Goldfarb L, Duchemann B, Chouahnia K, et al. Monitoring anti-PD-1-based immunotherapy in non-

Cite this article as: Evangelista L. The prediction of response to immunotherapy in non-small cell lung cancer patients by 18F-FDG PET/CT. J Thorac Dis 2019;11(11):E221-E223. doi: $10.21037 /$ jtd.2019.10.19 small cell lung cancer with FDG PET: introduction of iPERCIST. EJNMMI Res 2019;9:8.

6. Jreige M, Letovanec I, Chaba K, et al. 18F-FDG PET metabolic-to-morphological volume ratio predicts PDL1 tumour expression and response to PD-1 blockade in non-small-cell lung cancer. Eur J Nucl Med Mol Imaging 2019;46:1859-68.

7. Garg AD, Agostinis P. Cell death and immunity in cancer: from danger signals to mimicry of pathogen defense responses. Immunol Rev 2017;280:126-48.

8. Koh YW, Lee SJ, Park SY. 18F-Fluorodeoxyglucose positron emission tomography is correlated with the pathological necrosis and decreased microvessel density in lung adenocarcinoma. Ann Nucl Med 2019;33:93-102.

9. Shindo Y, Hazama S, Tsunedomi R, et al. Novel Biomarkers for Personalized Cancer Immunotherapy. Cancers (Basel) 2019. doi: 10.3390/cancers11091223. 